\title{
Role of a Preorganized Scaffold Presenting Four Residues of a GM-3 Lactone Mimetic on Melanoma Progression
}

\author{
Barbara Richichi, ${ }^{\dagger}$ Giuseppina Comito, ${ }^{\dagger}$ Olivier Renaudet, ${ }^{\S}, \|$ Michele Fiore, ${ }^{\S} \triangleleft$ A. Marra, ${ }^{\perp}$ B. Stecca, ${ }^{\#}$ \\ L. Pasquato, ${ }^{\nabla}$ P. Chiarugi, ${ }^{\ddagger}$ and C. Nativi* $*,, O$ \\ ${ }^{\dagger}$ Department of Chemistry "Ugo Schiff", University of Florence, via della Lastruccia, 1350019 Sesto F.no (FI), Italy \\ ${ }^{\ddagger}$ Department of Experimental and Clinical Biomedical Sciences, Biochemistry, Human Health Medical School, University of Florence, \\ viale Morgagni 50, 50134 Firenze, Italy \\ ${ }^{\S}$ Université Grenoble-Alpes, and CNRS, DCM, 38000 Grenoble, France \\ "Institut Universitaire de France, 103 Boulevard Saint-Michel, 75005 Paris, France \\ ${ }^{\perp}$ Institut des Biomolécules Max Mousseron (IBMM), UMR 5247, Ecole Nationale Supérieure de Chimie de Montpellier, 8 Rue de \\ l'Ecole Normale, 34296 Montpellier cedex 5, France \\ ${ }^{\#}$ Core Research Laboratory, Istituto Toscano Tumori, viale Pieraccini, 6, 50139 Firenze, Italy \\ ${ }^{\nabla}$ Department of Chemical and Pharmaceutical Sciences, University of Trieste, via L. Giorgieri 1, 34127 Trieste, Italy \\ FiorGen, Polo Scientifico e Tecnologico, via Sacconi, 650019 Sesto F.no (FI), Italy
}

Supporting Information

\begin{abstract}
Two tetravalent architectures, the glycocalix 7 and the RAFT 9, presenting four residues of a GM-3 ganglioside lactone mimetic, target the host compartment of melanoma and significantly abrogate the effect induced by cancer-associated fibroblasts (CAFs) contact + hypoxia in the motility and invasiveness of tumor cells. The data reported support the involvement of glycosphingolipids (GSLs) in hypoxia and show an interesting role played by compound 9 in targeting melanoma cells thereby interfering with melanoma progression. The unprecedented findings reported for the glycocluster 9 may contribute to the understanding of the critical and complex interactions between tumor cells and their local environment paving the way for new therapeutic agents.
\end{abstract}

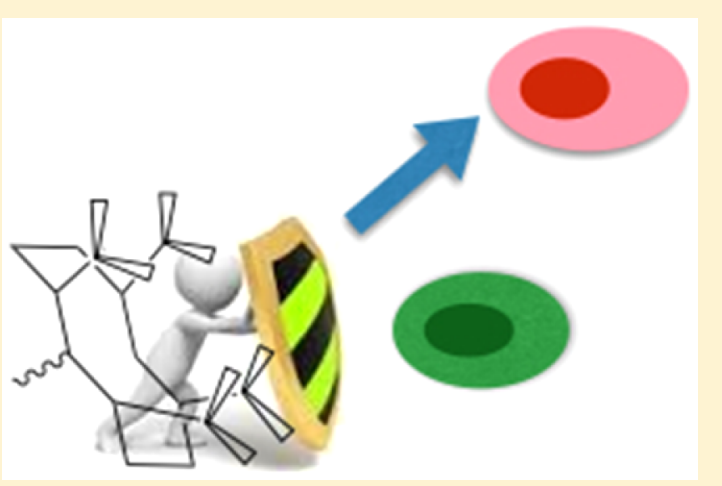

KEYWORDS: Glycosides, mimetics, tumor microenvironment, tumor antigen

$\mathrm{F}$ or many years neoplastic cells represented the focus of interest in cancer research. Nowadays, it is worldwide recognized that an important interplay exists between cancer cells and their local environment that plays a pivotal role in cancer "malignancy" (i.e., cancer cells' survival, adhesion, and motility). Thus, during primary tumor formation, cancer cells engage multifold interactions with an array of mesenchymal cells, including fibroblasts, macrophages, and endothelial cells. ${ }^{1}$ In addition, the tumor microenvironment (TME) evolves during cancer progression because cancer-associated fibroblasts (CAFs) are converted into myofibroblasts by cancer cellreleased factors; endothelial precursor cells are recruited from bone-marrow progenitors to facilitate de novo vasculogenesis, and inflammatory cells are converted and "educated" to behave as pro-tumoral cells. ${ }^{2}$ All these host cells engage a continuous molecular cross talk with cancer cells, secreting large amounts of factors/cytokines, influencing invasion and metastasis, ${ }^{2,3}$ and also enhancing resistance to apoptosis/anoikis and survival in an hostile environment characterized by hypoxia, acidity, and inflammatory cytokines delivery. ${ }^{4,5}$
In this framework, in the last two decades a great attention was focused on cancer matrix's composition, indeed therapies targeting the TME have begun to produce a number of agents that specifically target interactive pathways between tumor cells and the surrounding extracellular matrix. ${ }^{6-8}$

Glycosphingolipids (GSLs) and gangliosides in particular are known to interact with the key trans membrane receptors or signal transducers involved in cell adhesion and signaling thus affecting cellular phenotype. Though most studies considered the presence of GSLs on cells membranes only as possible markers, paying little attention to the functional role of these glycosyl epitopes in microdomains, ${ }^{9-11}$ at present strong evidence led to assume that the molecular mechanism of cancer "malignancy", reflecting enhanced cell motility with altered cell adhesion and growth, is based on how GSLs, and

Received: July 15, 2015

Accepted: November 10, 2015

Published: November 10, 2015 
particularly GM-2 and GM-3, are organized and assembled in membranes.

The understanding of GSLs-mediated mechanisms in cancer progression could certainly lead to the identification of novel targets for anticancer therapy. However, their scarce availability as pure compounds or their in vivo instability represent serious limiting steps. This has been the case of GM-3 ganglioside 1, the major ganglioside in normal melanocytes, which is overexpressed in melanoma cells with metastatic potential; likely due to the lower $\mathrm{pH}$ that characterizes tumor cells, $\mathbf{1}$ is transformed into the corresponding GM-3 lactone $2,{ }^{9-12}$ which has been recognized as the true antigen of melanoma (Figure 1 ). The scarce availability of GM-3 and particularly of GM-3

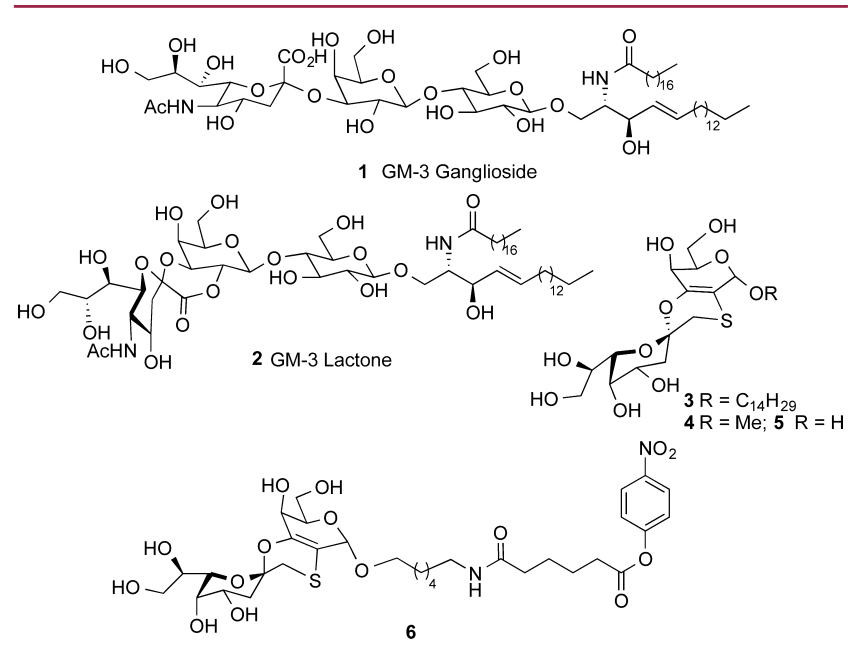

Figure 1. Structure of GM-3 ganglioside 1, GM-3 lactone 2, thioethers 3-5, and of the glycosyl derivative 6 .

lactone, their structure complexity and the instability of GM-3 lactone under physiological conditions, have severely limited a thorough investigation of their undoubted role in tumor malignancy and progression.

Following our interest in the high biological potential of GM3 and GM-3 lactone antigens, we recently designed and synthesized a mimetic of the GM-3 lactone, namely, the thioether 3 (Figure 1). ${ }^{13}$ From the structural point of view, 3 is simpler than its endogenous counterpart, is stable under physiological conditions, and maintains the characteristic folded shape of the native GM-3 lactone. Taking advantage of the remarkable stability and the versatility of the tricyclic structure, the precursor $\mathbf{5}$ was readily transformed into glycosyl derivatives such as the glycoconjugate 6 (see Figure 1), ${ }^{14,15}$ which was conveniently employed to give rise to multivalent glycoconjugates $^{15,16}$ (see Figure S1).

The effects of these glyco-derivatives on some key properties of malignant melanoma cells were preliminary assayed. ${ }^{16}$ The results showed that multivalency and spatial organization are essential features of the tested structures and indicated that the tetravalent glycocalix 7 possess an appropriate scaffold to interfere in melanoma cells progression and spreading (Figure 2).

In this context, following our preliminary data, we report herein the synthesis and biological tests of a new tetravalent cyclic glycopeptide, the glycosyl RAFT 9 (Scheme 1), which displays four residues of the glycomimetic $\mathbf{5}$ (see Figure 1 ) on the upper rim of the cyclic platform ( $\mathbf{R}$, see Scheme 1). Globally, in the constructs 7 and 9 the four epitopes are

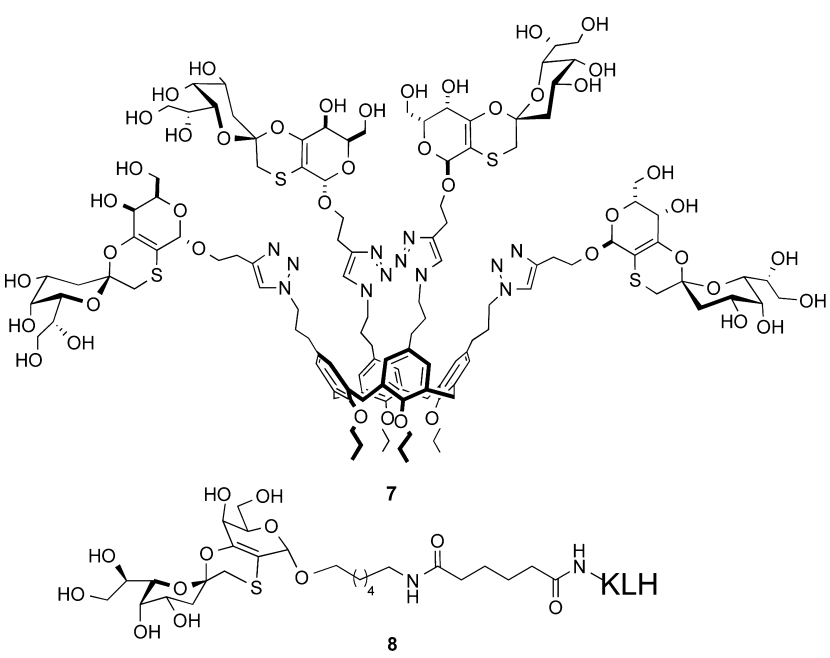

Figure 2. Structure of the glycocalix 7 and of the KLH-glycoconjugate 8.

Scheme 1. Synthesis of the RAFT 9

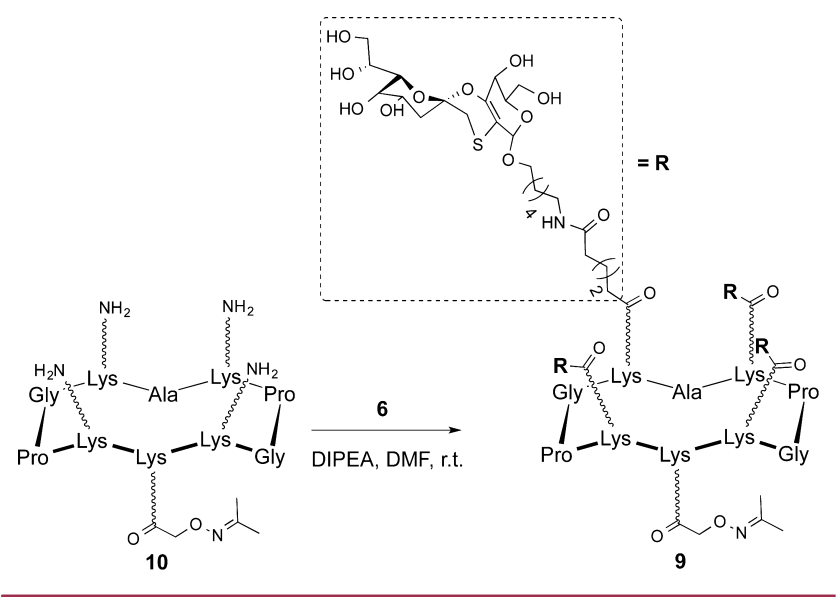

roughly located in the same spatial frame, although the chemical nature and the biocompatibility of the two scaffolds are substantially different.

Keeping in mind the reported activity of the GM-3 mimetic on melanoma progression, ${ }^{16}$ the effect of the RAFT 9 (see Scheme 1) on melanoma cell adhesion, migration, and resistance to apoptosis (anoikis) was assayed to investigate any additional extra effect, favorable or detrimental played by the scaffold, in order to obtain a more versatile and efficient melanoma modulator. Furthermore, the RAFT 9 and, for comparison, the glycocalix 7 , were tested in events affected by the microenvironment and of significance in tumor progression. Finally, taking into account the interesting results obtained with the KLH-glycoconjugate 8 (see Figure 2) as immunostimulant, ${ }^{15}$ the glycosyl RAFT 9 was also assayed in vivo.

Synthesis of the RAFT-Based Glycocluster 9. Cyclopeptides have been proved useful scaffolds for the synthesis of antitumoral vaccines. ${ }^{17}$ Beside their stable conformation in solution and improved resistance in vivo, RAFT (Regioselectively Addressable Functionalized Templates) structures have the advantage of presenting regioselectively addressable domains on which diverse antigens can be covalently attached in a well-defined spatial orientation, thus avoiding steric hindrance among them. ${ }^{18}$ In particular, four-component vaccine candidates have been prepared with good yields, 
excellent molecular definition and remarkable immunological profile. ${ }^{19-21}$ For these reasons, the RAFT scaffold appeared to be an attractive system for the present study. The RAFT-based glycocluster 9 (see Scheme 1) was prepared by coupling the RAFT scaffold $10^{18}$ with the glycosyl derivative 6 . The reaction was performed under mild conditions (r.t. and DIPEA as base) and in DMF as solvent. Compound 9 was isolated in $77 \%$ yield by column chromatography on Sephadex LH-20 (Scheme 1).

Biological Assays in Vitro. The effect of the RAFT-based glycocluster 9 on melanoma cell adhesion, migration, and resistance to apoptosis (anoikis) was assayed as previously described. ${ }^{16}$ In addition to migration, we investigated the effect of compound 9 on cells ability to cross 3D barrier using Boyden chambers with $8 \mu \mathrm{m}$ pores in the presence of a $3 \mathrm{D}$ barrier of Matrigel. A375 invasiveness toward 10\% serum was allowed for $24 \mathrm{~h}$ before counting the cells having crossed the filter. We then analyzed the effect of compounds 7 and 9 on TME. We used $1 \% \mathrm{O}_{2}$ hypoxia and contact with CAFs, two key features playing a synergetic role for secretion of proinflammatory and pro-angiogenic cytokines during melanoma invasiveness. Human melanoma A375 cells were treated with conditioned media from CAFs in hypoxia in the presence of 7 or 9 and assayed for their ability to migrate or to invade through reconstituted matrigel barrier.

As shown in Figure 3, compound 9 was significantly active on several key hallmarks of malignant melanoma cells. Specifically, it was able to inhibit to an extent up to $30 \%$ the adhesion of melanoma cells on fibronectin matrices and its inhibitory activity grew up to $50 \%$ after $4 \mathrm{~h}$ of incubation (Figure $3 \mathrm{~A}$ ). Moreover, compound 9 induced a doubling of spontaneous apoptotic death of treated melanoma cells. Concerning the
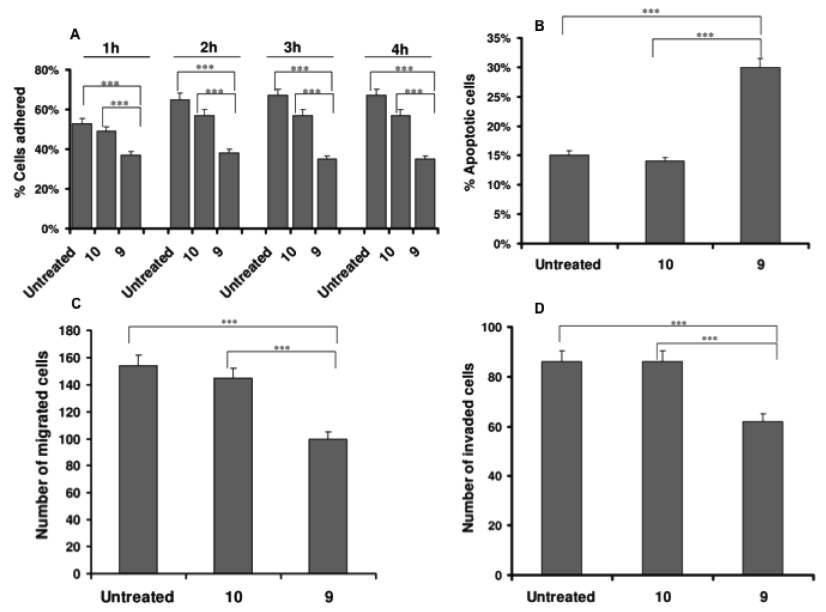

Figure 3. (A) A375 cells were treated with $50 \mu \mathrm{M}$ of RAFT 9 and left to adhere onto fibronectin-coated culture dishes for different times. The percentage of adhered cells, in the presence or in the absence of fibronectin, was evaluated by crystal violet assay. (B) Cells were serum starved, detached, and kept in suspension for $24 \mathrm{~h}$ in the presence of RAFT 9. The percentage of apoptotic cells was evaluated using the Annexin V-Pi staining Kit. (C) Migration assay. Cells treated as in panel A were allowed to migrate for $24 \mathrm{~h}$ toward $10 \%$ serum as chemo attractant. For each filter the number of cells in the randomly chosen field was determined. (D) Invasiveness assays. Invasiveness was evaluated as for the migration assay B except that matrigel has been used to represent 3D ECM. All data are representative of at least three independent experiments, and means and SD are shown in the graphs. RAFT 10 was tested as negative control: $* * *, P<0.001$ versus each correspondent control. activity of 9 on the motility of melanoma cells (Figure 3B), we observed a $30 \%$ reduction of both $3 \mathrm{D}$ cell migration and invasiveness across tissue barriers (Figure 3C,D).

Tumor cells do not grow and progress toward malignancy alone, but coevolve together with a number of accessory populations, among which the most representative are CAFs. Moreover, several structural cues affect the motility of cancer cells, of which hypoxia and the consequent acidity are the most relevant. We have recently reported that in melanoma cells the contact with CAFs, known to induce in other cancer models a motility stimulus through activation of an epigenetic program called epithelial mesenchymal transition, needs the concomitant presence of hypoxic treatments. ${ }^{22}$ Therefore, we treated melanoma cells with the conditioned medium of cancerassociated cells (derived ex vivo from human aggressive melanoma) and analyzed the effects of compounds 7 and 9. Our results indicated that both 7 and 9 were able to exert a very strong effect on both 2D migration and invasiveness of melanoma cells, completely abrogating the effects exerted by tumor microenvironment (CAFs + hypoxia) (Figures 4A,B).

Biological Assays in Vivo. In the past decade, the chemical and biological properties of RAFT cyclopeptides have been extensively investigated, ${ }^{18}$ and RAFT scaffolds have widely been used to present saccharidic epitopes to the immune system. ${ }^{19-21}$ Analogously, the KLH-glycoconjugate 8 (see Figure 2) that presents residues of the mimetic $\mathbf{5}$ (see Figure
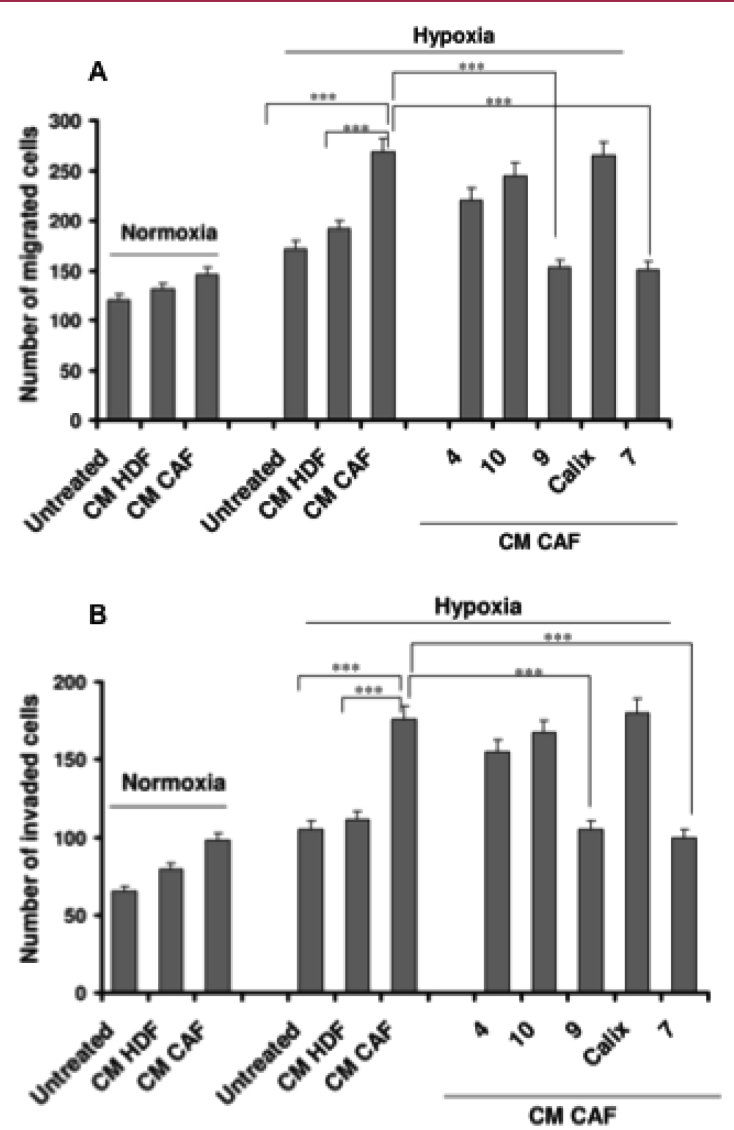

Figure 4. A375 cells were incubated in hypoxia with CM from HDFs or CAFs, in the presence or in the absence of the tested compounds $(50 \mu \mathrm{M})$. Migration (A) or invasion (B) was thus evaluated. The monovalent mimetic 4, the Calix (see Figure S1), and the RAFT 10 were tested as negative controls; ***, $P<0.001$ versus each correspondent control. 
1) proved to elicit in mice a specific immunoresponse against GM-3 overexpressed on melanoma cells. ${ }^{15}$ These captivating data encouraged us to test the RAFT-based glycoconjugate 9 in vivo. On days $0,14,28,49$, and 72 three groups of ten C57BL/ 6 mice were immunized i.p. with $400 \mu \mathrm{g}$ of compound 9, 4Lys1Melan [i.e., R(4Lys-1Ox)], and PBS (control group), and B16F10 cells were injected at day 69. Our results show that compound 9 was able to reduce by more than $50 \%$ the tumor volume of B16F10 xenografts compared to PBS control ( $p<$ $0.001)$. On the contrary, tumor growth in mice treated with 4Lys-1Melan was not statistically different from that of mice treated with PBS (Figure 5). Unfortunately, the short-term in vivo experiment prevented us from assessing formation of lung or liver metastases.

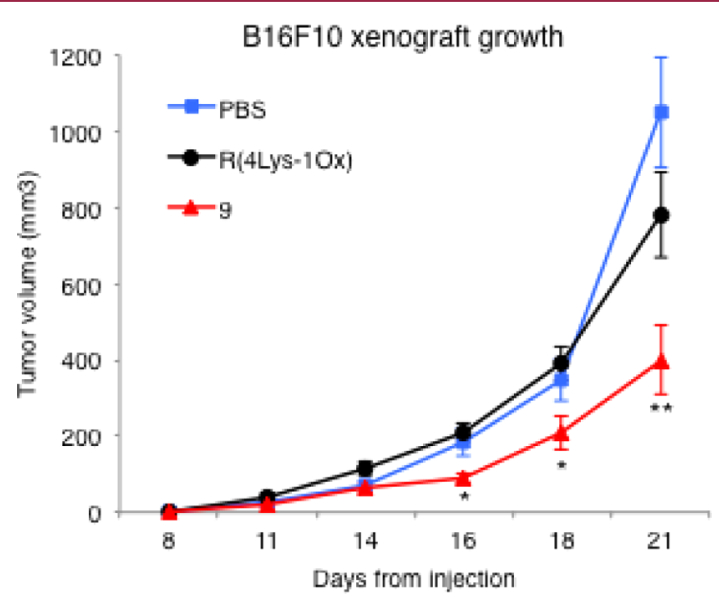

Figure 5. Effect of RAFT 9 and R(4Lys-1Ox) on in vivo tumor growth. Three groups of C57BL/6 mice (10 mice per group) were treated with 9, R(4Lys-1Ox), and PBS (as negative control) on days $0,14,28,49$, and 72 (doses: $400 \mu \mathrm{g}$; all i.p.). On day 69, 10,000 B16F10 murine melanoma cells were injected subcutaneously in two lateral flanks. The volume of the tumor (20 tumors per group) was measured every 2-3 days with a caliper, and tumor volumes were calculated with the formula $V=W^{2} \times L \times 0.5$, where $W$ and $L$ are tumor width and length, respectively. Data are presented as mean \pm SEM $*, p<0.05$; **, $p<0.001$ versus each correspondent PBS control.

A clear evidence of the complex interplay between cancer cells and their local environment can be found in the data reported above. As a matter of fact, the results described in Figure 3 indicate that compound 9 is an effective inhibitor of melanoma adhesive properties. In particular, compound 9 inhibits both migration and invasiveness of melanoma cells, two features of malignant cancer cells extremely "useful" to invade surrounding tissues, evade from primary tumor environment, and propagate a metastatic colony elsewhere. A minor contribution seems to be played by the peptide skeleton of the molecule ruling out a significant interference from the scaffold 10.

Compound 9 is also active in inducing apoptotic death due to anoikis, a particular programmed cell death caused by the lack or improper integrin-mediated cell anchorage. Several cancer cells, including malignant melanoma, exploit resistance to natural anoikis program in blood or lymphatic circulation, to survive and spread to distant organs.

The ability of compound 9 to inhibit migration, invasion, and survival in the absence of proper integrin-mediated adhesion, thereby inducing apoptosis, shows that 9 exerts a wide activity toward several features of melanoma malignancy.
As tumor cells are greatly helped in gaining malignant attitudes by TME factors, we also studied the effects of compound 9 on melanoma cell motility and invasiveness upon exposure to hypoxia and contact with stromal fibroblasts. Since we have recently reported that compound 7 affects motility and invasiveness of melanoma cells, ${ }^{16}$ though in a lesser extent with respect to 9, we also included compound 7 in the TME experiments.

Due to deficiency or lack of vasculature in tumors, hypoxia is a very common feature of several neoplasiae. This causes a deprivation of oxygen and nutrients, impairing the survival of cancer cells but also stimulating pro-invasive properties of malignant cells. In addition to hypoxia, several cancers are highly infiltrated by CAFs. Altogether, they form the tumor reactive stroma. ${ }^{23,24}$

CAFs have been recognized to play an essential role in activating a motile and survival epigenetic program in several models of cancers. ${ }^{25}$ As above-mentioned, for melanoma cells, hypoxia and CAFs exert a synergistic role in regulating motility and invasiveness of malignant melanoma cells. ${ }^{22}$ We now observed that the exposure of melanoma cells to hypoxia alone did not elicit a strong increase in A375 melanoma cell invasive and motile phenotype, but the concomitant treatment with conditioned medium from CAFs gave rise to a strong increase in aggressiveness. Worth noting, both compounds 7 and 9 were extremely active in abrogating the effect induced by CAFs contact + hypoxia in melanoma cells motility and invasiveness (Figure 4), whereas resulted ineffective during the treatment with hypoxia only (data not shown). These observations are perfectly in line with the hypothesis that CAFs sensitize malignant melanoma cells to hypoxia, raising interest in compounds 7 and 9 to target the Achilles's heel of the tumor vs stroma.

The ability of 7 and 9 to regulate stromal reactivity and tumor microenvironment features may also account for a potential inhibitory effect, on melanoma growth in vivo, which may be ascribed to the reshaping of microenvironment and stromal activation. Thus, the bioinspired peptide 9 was also tested in vivo to investigate the ability of targeting cancer cells in addition to TME. Compound 9 was thus used to treat C57BL/ 6 mice, and the growth of induced subcutaneous tumor was monitored. The reduction of tumor growth observed in mice treated with 9 with respect to those treated with PBS (negative control) is remarkable. This unprecedented feature makes the compound 9 a sort of Janus synthetic antigen, targeting melanoma cells as well as their microenvironment. Further experiments aimed at understanding the mechanisms of action of the RAFT-glycocluster 9 are currently in progress.

In conclusion, tumor microenvironment plays a dramatic role in cancer progression endowing cancer cells with malignant properties culminating in metastasis. This is actually the major cause of death in cancer patients. In this Letter, we reported the effects of local environment on melanoma cell changes and aggressiveness. We showed that two tetravalent compounds, namely, 7 and 9, could abrogate the "malignant" effects induced by CAFs contact + hypoxia on melanoma cells, significantly reducing their mobility and invasiveness, that is their metastatic potential. In particular, the biological results confirmed (i) the unquestionable, though elusive, role of GSLs in cancer progression, (ii) the puzzling influence of microenvironment in tumor aggressiveness and invasion, and (iii) the biological activity of the thioether mimetic of the GM-3 lactone 2. In vivo tests pointed out that $\mathbf{9}$ is also active in reducing tumor onset, 
suggesting an unprecedented dual role of 9 in melanoma modulation. Although a complete rationale of the multifaceted cancer's onset and growth still lag far behind, we believe that our findings might give a contribution to the development of new therapies targeting tumor cells and the microenvironment.

\section{EXPERIMENTAL PROCEDURES}

Synthesis of Compound 9. To a stirred solution of $\mathbf{1 0}(9.0 \mathrm{mg}$, $0.0056 \mathrm{mmol})$ in dry DMF $(1.8 \mathrm{~mL})$, DIPEA $(5.0 \mu \mathrm{L}, 0.026 \mathrm{mmol})$ was added $(\mathrm{pH}=8)$. The mixture was stirred for $10 \mathrm{~min}$ at $\mathrm{rt}$, then a solution of $\mathbf{5}(30.0 \mathrm{mg}, 0.041 \mathrm{mmol})$ in dry DMF $(3.0 \mathrm{~mL})$ was added. The reaction mixture was followed by LC-MS (Column: AgilentZorbax 300SB-C18, $4.6 \times 250 \mathrm{~mm}$. Eluant: AcCN 90\%/ $\mathrm{H}_{2} \mathrm{O}+0.1 \%$ TFA from 5:95 to 100 in $15 \mathrm{~min} ; 10 \mathrm{rt} 6.60 \mathrm{~min}$ ) monitoring the complete disappearance of 10 . After $3.0 \mathrm{~h}$ at $\mathrm{rt}$ the reaction mixture was concentrated to dryness. The crude product was purified on Sephadex LH-20 to give $15 \mathrm{mg}$ of 9 as white glassy solid (77\%). ${ }^{[\alpha]} \mathrm{D}_{25}$ +29.1 (c 0.4 in $\left.\mathrm{CH}_{3} \mathrm{OH}\right)$. HRMS $m / z$ : calcd. for $\mathrm{C}_{156} \mathrm{H}_{258} \mathrm{O}_{60} \mathrm{~N}_{2} \mathrm{OS}_{4}$ $[\mathrm{M}+2 \mathrm{H}]^{+} 1749.83120$, found 1749.82642 .

\section{ASSOCIATED CONTENT}

\section{(S) Supporting Information}

The Supporting Information is available free of charge on the ACS Publications website at DOI: 10.1021/acsmedchemlett.5b00283.

Materials and methods; structures of PAMAM-based glycocluster, glycosyl gold nanoparticles, Calix, and 4Lys1Melan; plot of cells viability test; tests on HS29 4T and $\mathrm{HDF} ; \mathrm{EC}_{50}$ (PDF)

\section{AUTHOR INFORMATION}

\section{Corresponding Author}

*E-mail: cristina.nativi@unifi.it.

\section{Present Address}

Université de Lyon, 92, rue Pasteur; CS 30122, Lyon Cedex 07, France.

\section{Author Contributions}

C.N., P.C., O.R., B.R., and B.S. designed research; B.R., G.C., and M.F. performed research; C.N., P.C., O.R., B.R., B.S., A.M., and L.P. analyzed data, and C.N. and P.C. wrote the paper. All authors have given approval to the final version of the manuscript.

\section{Funding}

C.N., P.C., B.R., and G.C.: AIRC (Associazione Italiana Ricerca sul Cancro, IG 2012), Ente Cassa di Risparmio di Firenze and COST action CM1102 for financial support. O.R.: "Communauté d'agglomération Grenoble-Alpes Métropole” (Nanobio program), the ICMG FR 2607. O.R and M.F.: the Ligue contre le cancer (MF), the Agence Nationale de la Recherche Grant ANR-12-JS07-0001-01 "VacSyn", and the Labex ARCANE (ANR-11-LABX-0003-01).

\section{Notes}

The authors declare no competing financial interest.

\section{ACKNOWLEDGMENTS}

Authors are grateful to Prof. S.-i. Hakomori for his inspiring work on GM-3.

\section{ABBREVIATIONS}

CAFs, cancer-associated fibroblasts; GSLs, glycosphingolipids; TME, tumor microenvironment; HDFs, human dermal fibroblasts; KLH, keyhole lymphocyte hemocyanine

\section{REFERENCES}

(1) Mueller, M. M.; Fusenig, N. E. Friends or foes bipolar effects of the tumour stroma in cancer. Nat. Rev. Cancer 2004, 4, 839-49.

(2) Kalluri, R.; Zeisberg, M. Fibroblasts in cancer. Nat. Rev. Cancer 2006, 6, 392-401.

(3) Lorusso, G.; Ruegg, C. Histochem. The tumor microenvironment and its contribution to tumor evolution toward metastasis. Histochem. Cell Biol. 2008, 130, 1091-103.

(4) Taddei, M. L.; Giannoni, E.; Comito, G.; Chiarugi, P. Microenvironment and tumor cell plasticity: an easy way out. Cancer Lett. 2013, 341, 80-96.

(5) Pani, G.; Galeotti, T.; Chiarugi, P. Metastasis: cancer cell's escape from oxidative stress. Cancer Metastasis Rev. 2010, 29, 351-78.

(6) Sounni, N. E.; Noel, A. Targeting the tumor microenvironment for cancer therapy. Clin. Chem. 2013, 59, 85-93.

(7) Friedl, P.; Alexander, S. Cancer invasion and the microenvironment: plasticity and reciprocity. Cell 2011, 147, 992-1009.

(8) Swartz, M. A.; Iida, N.; Roberts, E. W.; Sangaletti, S.; Wong, M. H.; Yull, F. E.; Coussens, L. M.; DeClerck, Y. A. Tumor microenvironment complexity: emerging roles in cancer therapy. Cancer Res. 2012, 72, 2473-80.

(9) Hakomori, S.-i. The glycosynapse. Proc. Natl. Acad. Sci. U. S. A. 2002, 99, 225-32.

(10) Hakomori, S.-i. Glycosynaptic microdomains controlling tumor cell phenotype through alteration of cell growth, adhesion, and motility. FEBS Lett. 2010, 584, 1901-6.

(11) Sonnino, S.; Prinetti, A. Sphingolipids and membrane environments for caveolin. FEBS Lett. 2009, 583, 597-606.

(12) Harada, Y.; Sakatsume, M.; Nores, G. A.; Hakomori, S.-i.; Taniguchi, M. Density of GM3 with normal primary structure determines mouse melanoma antigenicity; a new concept of tumor antigen. Jpn. J. Cancer Res. 1989, 80, 988-92.

(13) Singhal, A.; Hakomori, S.-i. Molecular changes in carbohydrate antigens associated with cancer. BioEssays 1990, 12, 223-8.

(14) Toma, L.; Di Cola, E.; Ienco, A.; Legnani, L.; Lunghi, C.; Moneti, G.; Richichi, B.; Ristori, S.; Dell'Atti, D.; Nativi, C. Synthesis, conformational studies, binding assessment and liposome insertion of a thioether-bridged mimetic of the antigen GM3 ganglioside lactone. ChemBioChem 2007, 8, 1646-9.

(15) Arcangeli, A.; Toma, L.; Contiero, L.; Crociani, O.; Legnani, L.; Lunghi, C.; Nesti, E.; Moneti, G.; Richichi, B.; Nativi, C. Stable GM3 lactone mimetic raises antibodies specific for the antigens expressed on melanoma cells. Bioconjugate Chem. 2010, 21, 1432-36.

(16) Richichi, B.; Comito, G.; Cerofolini, L.; Gabrielli, G.; Marra, A.; Moni, L.; Pace, A.; Pasquato, L.; Chiarugi, P.; Dondoni, A.; Toma, L.; Nativi, C. Multivalent presentation of a hydrolytically stable GM3 lactone mimetic as modulator of melanoma cells motility and adhesion. Bioorg. Med. Chem. 2013, 21, 2756-63.

(17) Renaudet, O.; BenMohamed, L.; Dasgupta, G.; Bettahi, I.; Dumy, P. Towards a self-adjuvanting multivalent $\mathrm{B}$ and $\mathrm{T}$ cell epitope containing synthetic glycolipopeptide cancer vaccine. ChemMedChem 2008, 3, 737-741.

(18) Galan, M. C.; Dumy, P.; Renaudet, O. Multivalent glyco(cyclo)peptides. Chem. Soc. Rev. 2013, 42, 4599-4612.

(19) Renaudet, O.; Dasgupta, G.; Bettahi, I.; Shi, A.; Nesburn, A. B.; Dumy, P.; BenMohamed, L. Linear and branched glyco-lipopeptide vaccines follow distinct cross-presentation pathways and generate different magnitudes of antitumor immunity. PLoS One 2010, 5, e11216.

(20) Grigalevicius, S.; Chierici, S.; Renaudet, O.; O-Man, R.; Dériaud, E.; Leclerc, C.; Dumy, P. Chemoselective assembly and immunological evaluation of multiepitopic glycoconjugates bearing clustered Tn antigen as synthetic anticancer vaccines. Bioconjugate Chem. 2005, 16, 1149-1159.

(21) Richichi, B.; Thomas, B.; Fiore, M.; Bosco, R.; Qureshi, H.; Nativi, C.; Renaudet, O.; BenMohamed, L. A cancer therapeutic vaccine based on clustered Tn-antigen mimetics induces strong antibody-mediated protective immunity. Angew. Chem., Int. Ed. 2014, 53, 11917-20. 
(22) Comito, G.; Giannoni, E.; Di Gennaro, P.; Segura, C. P.; Gerlini, G.; Charugi, P. Stromal fibroblasts synergize with hypoxic oxidative stress to enhance melanoma aggressiveness. Cancer Lett. 2012, 324, $31-41$.

(23) Cirri, P.; Chiarugi, P. Cancer-associated-fibroblasts and tumour cells: a diabolic liaison driving cancer progression. Cancer Metastasis Rev. 2012, 31, 195-208.

(24) Barcellos de Sousa, P.; Gori, V.; Bambi, F.; Chiarugi, P. Tumor microenvironment: bone marrow-mesenchymal stem cells as key players. Biochim. Biophys. Acta, Rev. Cancer 2013, 1836, 321-35.

(25) Parri, M.; Chiarugi, P. Redox molecular machines involved in tumor progression. Antioxid. Redox Signaling 2013, 19, 1828-45. 